\section{ORIGINAL RESEARCH}

S. Merino

J. Arrazola

A. Cárdenas

M. Mendoza

P. De Miguel

C. Fernández

T. Ganado

\title{
Utility and Interobserver Agreement of Ultrasound Elastography in the Detection of Malignant Thyroid Nodules in Clinical Care
}

\begin{abstract}
BACKGROUND AND PURPOSE: Malignancy correlates with hardness of tissues and US elastography can potentially analyze the stiffness of lesions. Our aim was to evaluate the utility of US elastography in the detection of malignant nodules and to investigate interobserver agreement with this technique.

MATERIALS AND METHODS: One-hundred three consecutive patients with 106 thyroid nodules were examined prospectively with conventional B-mode sonography and real-time US elastography. All patients were referred for FNAB. Conventional B-mode sonography and US elastographic examinations were performed, and images were separated and independently interpreted by 2 radiologists blinded to pathologic results. US elastogram evaluation was based on a simplified classification of stiffness based on gray-scale patterns, tumor size compared with B-mode, and margins. Interobserver agreement was studied. FNAB was used as the reference standard for the diagnosis of benign nodules, but histopathologic evaluations were performed when results suspicious for malignancy or malignant results were obtained on FNAB as well as in indeterminate lesions.
\end{abstract}

RESULTS: In our study, pattern of stiffness based on gray-scale and classification proposed were statistically significant and predicted malignancy with $100 \%$ sensitivity and $40.6 \%$ specificity. Tumor size when compared with B-mode images or margins was not statistically significant in our study. No false-negatives were found, and an NPV of $100 \%$ was seen. Interobserver agreement for US elastography was excellent in our study, with a $\kappa$ index of $0.82(95 \% \mathrm{Cl})$.

CONCLUSIONS: We believe that US elastography is a promising technique that can assist in the evaluation of thyroid nodules and can potentially diminish the number of FNAB procedures needed. We believe that it may be useful to introduce US elastography into routine clinical practice.

ABBREVIATIONS $\mathrm{Cl}=$ confidence interval; FNAB = fine-needle aspiration biopsy; NPV = negative predictive value; $\mathrm{PPV}=$ positive predictive value; $\mathrm{QF}=$ quality factor; $\mathrm{US}=$ ultrasound

$T^{1}$ hyroid nodules are found in $4 \%-8 \%$ of adults by palpation, $41 \%$ by sonography, and $50 \%$ by pathologic postmortem examination. ${ }^{1}$ Although in the general population, most thyroid nodules are benign, the prevalence of thyroid cancer is as high as $5 \%-10 \% .{ }^{1}$ Papillar carcinoma represents $75 \%-80 \%$ of the cases, and other histologic types include follicular carcinoma (10\%-20\%), medullar carcinoma (3\%$5 \%)$, and anaplastic carcinoma (1\%-2\%). Lymphoma and metastases of the thyroid gland are less frequent.

The management of thyroid nodules is of clinical importance due to the relatively high incidence of malignant disease and the relative difficulty in distinguishing benign from malignant nodules by conventional sonographic imaging. ${ }^{2-4}$

At the moment, conventional sonographic images remain the best way to delimitate thyroid nodules and to determine which nodules should be studied by FNAB because of suspicion for malignancy. ${ }^{5,6}$ Despite being extremely accurate, conventional sonography is not as useful for differentiating benign and malignant nodules. Conventional sonographic signs that correlate with malignant lesions include the following: hypoechogenicity, blurred or irregular margins, microcalcifi-

Received February 3, 2011; accepted after revision April 3.

From the Departments of Radiology (S.M., J.A., A.C., T.G.), Pathology (M.M.), Endocrinology (P.D.M.), and Preventive Medicine (C.F.), Hospital Clínico San Carlos, Madrid, Spain.

Please address correspondence to: Salomé Merino, MD, Department of Radiology, Hospital Clínico San Carlos, Universidad Complutense of Madrid, Martín Lagos s/n. 28040 Madrid, Spain; e-mail: salome.merino@yahoo.es

http://dx.doi.org/10.3174/ajnr.A2716 cations, an anteroposterior/transverse diameter of $\geq 1 \mathrm{~cm}$, and intranodular predominantly centric vascularity. ${ }^{7-9}$ However the sensitivity, specificity, NPV, and PPV are considerably variable from study to study. ${ }^{10-12}$

FNAB provides the best method to differentiate benign from malignant nodules. ${ }^{13}$ In most of the proposed guidelines, current management of thyroid nodules includes the FNAB procedure in all nodules with a diameter $>1 \mathrm{~cm}$ and in those with lesser diameters but with different classic sonographic signs suspicious for malignancy. US-guided FNAB has proved to be an efficient tool for thyroid cancer diagnosis, especially in the case of papillary carcinoma. In follicular carcinoma, histopathology is required because the diagnosis is based on the presence or absence of capsular or vascular invasion. ${ }^{14-16}$ However, US-guided FNAB is an invasive procedure, subject to sampling errors, and consumes time and economic resources, and it is associated with minor complications.

The high incidence of nodules, the invasiveness of the procedure, and the possibility of sampling error require performing protocols for determining with relative confidence which nodules should be followed up and which should be aspirated.

Malignant nodules tend to be much harder than benign ones. However, the stiffness of thyroid nodules in palpation is a nonobjective indicator for the differential diagnosis. US elastography has been developed to obtain information about tissue stiffness noninvasively. This technique can evaluate the degree of distortion of a tissue under application of an external force and is based on the principle that the softer parts of 


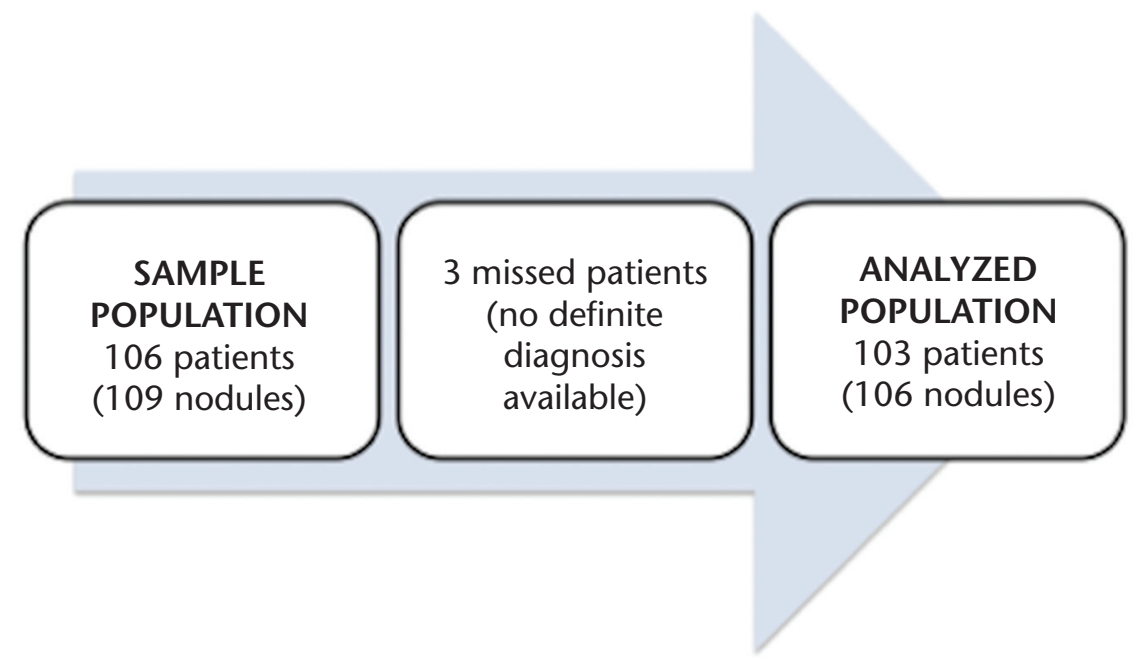

Fig 1. Flow chart of the study. Three patients were excluded from the sample population because no definite pathologic result was available. A total of 103 patients and 106 nodules were definitively included in the study (analyzed population)

tissues deform easier than the harder ones. ${ }^{17}$ US elastography has been successfully applied to breast lesions, the prostate, pancreas, lymph nodes, and thyroid gland, all easily assessable on elastographic examination. ${ }^{18-21}$

The aim of this prospective study was to find out possible elastographic signs suspicious for malignancy in thyroid nodules, to evaluate interobserver agreement for US elastography, and to explore the sensitivity and specificity of US elastography for the differential diagnosis of thyroid cancer, with pathologic analysis as the reference standard.

\section{Materials and Methods}

\section{Research Design}

The study design was based in a series of consecutive thyroid nodules evaluated with conventional B-mode sonography and US elastography between October 2008 and November 2009, with prospective capture of information.

\section{Patients}

One-hundred six consecutive patients who were referred for FNAB of thyroid nodules were initially included in this prospective study. In 3 of these patients, it was not possible to obtain pathologic results, so they were excluded from the final statistical study. The sample at FNAB was insufficient in 1 patient who left our country and could not be followed up. The cytologic result in another patient was atypia of uncertain significance in subsequent explorations, and the patient declined new FNAB or surgery. The third patient also declined surgery even with FNAB results suspicious for follicular neoplasia.

Finally, 103 patients ( 89 women and 14 men) were included. The mean age was 58 years (range, $21-87$ years). In each patient, 1 thyroid nodule was studied, except in 3 patients in whom 2 nodules were evaluated, so 106 nodules were definitively included in the study (Fig 1).

Before enrollment, each patient gave written informed consent.

\section{Equipment}

Conventional B-mode sonography and elastographic examinations were performed by using a real-time machine (Acuson S2000; Sie- mens, Erlangen, Germany) and a $13.5-\mathrm{MHz}$ linear probe. Specific software was used to measure tissue distortion.

\section{Imaging Methods}

Conventional Sonographic Examination. Initially, conventional sonographic images were obtained with the same method used for standard clinical thyroid gland explorations, with the neck slightly extended. Morphologic aspects of the nodule such as size $(<1 \mathrm{~cm} ; 1-2$ $\mathrm{cm}$; and $>2 \mathrm{~cm}$ ), echogenicity (hypoechoic, hyperechoic, isoechoic, heterogeneous), margins (regular/well-defined or irregular/ill-defined), presence or absence of calcifications (microcalcifications and coarse calcifications), presence or absence of cysts, acoustic transmission (bad, good, or indeterminate), and absence or presence (continuous or interrupted) of halo were evaluated.

The images were acquired, reviewed, and interpreted by 2 radiologists with 6 and 12 years' experience, respectively, in the evaluation of thyroid nodules. Both were blinded to the final pathologic diagnoses. When there was no agreement, consensus was obtained. It is not the purpose of this article to evaluate the utility of B-mode findings in the differential diagnosis of benign or malignant nodules, or even the interobserver agreement with this technique.

During B-mode sonography exploration, thyroid gland lesions were identified and the region of interest for elastography was selected.

US Elastography. After the B-mode sonographic examination, US elastography was performed. The same real-time instruments and probes were used. The probe was placed on the neck with light pressure, and the operator highlighted a box, which included the nodule and sufficient surrounding thyroid tissue when possible. The freehand compression applied on the neck region was slight, and an overlay box indicated too little/too much axial motion or excessive lateral motion. The application displayed a real-time QF score by using advanced signal-intensity analysis. A high QF value $(>60)$ indicated minimal global motion artifacts, and a low QF value $(<50)$ indicated global motion artifacts that may decrease diagnostic value.

The US-elastogram was displayed next to the B-mode image; grayscale images were registered and recorded for subsequent analysis.

As with conventional sonography, the 2 radiologists independently evaluated the different variables defined for US elastography 
analysis for each nodule. Moreover, when discrepancies occurred, consensus was obtained.

In terms of elastography, radiologists evaluated elasticity (predominantly rigid, indeterminate, and predominantly soft), size (larger diameter on US elastography than on B-mode sonography, similar size or smaller diameter on US elastography), and margins (ill-defined or irregular, indeterminate, or well-defined or regular). Elasticity was evaluated according to gray-scale elastograms: Lesions were considered predominantly rigid when $>90 \%$ of the area of the lesion was black and predominantly soft when $>90 \%$ area of the lesion was white in gray-scale; an indeterminate category was considered when lesions could not be included in any of these categories.

The best B-mode US elastographic image pairs were acquired and stored for posterior evaluation. Both images were physically separated to avoid the influence of one exploration over another.

According to variables described above and before consensus was reached, we established some criteria to let each radiologist classify nodules into 3 groups, first separately and then by consensus, depending on the suspicion for malignancy or benignity of the lesions with US elastography: type I (suspicious for benignity), type II (indeterminate), and type III (suspicious for malignancy). These criteria are as follows: Type I nodules (suspicious for benignity or predominantly soft): predominantly white in gray-scale elastograms ( $>90 \%$ of the area of the nodule); Type II nodules (indeterminate): classification in type I or III was not possible; Type III nodules (suspicious for malignancy or predominantly rigid): predominantly black in gray-scale elastograms ( $>90 \%$ of the area of the nodule) or greater size with US elastography than on the B-mode image.

\section{Histopathologic Diagnosis}

Cytologic or histologic diagnosis served as the reference standard for comparison between conventional sonographic imaging and US elastography.

According to cytologic results obtained with FNAB and the classification proposed by the Bethesda System for Reporting Thyroid Cytopathology, we classified thyroid nodules into 1 of the following groups: 1) malignant, 2) suspicious for malignancy, 3) suspicious for follicular or Hürthle cell neoplasia, 4) atypia of uncertain significance, 5) benign, and 6) insufficient or not valid material. ${ }^{20,21}$ In our hospital, the pathologist is always present when FNAB samples are obtained to reduce the amount of insufficient material or samples with atypia of uncertain significance; an insufficient amount or atypia requires repetition of the procedure.

A definite diagnosis was obtained with FNAB and cytology only in patients included in category 5 (benign). Patients with nodules included by FNAB in groups 1 (malignant) and 2 (suspicious for malignancy) were scheduled for surgery, and histologic results were analyzed. The same procedure occurred with nodules considered in category 3 (suspicious for neoplasia), in which surgery was needed to distinguish carcinoma and adenoma because cytology cannot differentiate these 2 entities. Nodules included in category 4 (atypia of uncertain significance) or 6 (insufficient or not valid material) were re-evaluated to reclassify them into other groups mentioned before.

Definite nodule pathologic classification from cytologic or histologic analyses established 2 groups: 1) malignant nodules, and 2) benign nodules.

\section{Statistical Analysis}

Qualitative variables were studied as percentages, and quantitative variables were analyzed as means and SDs. Parametric tests were used for statistical evaluation. Results obtained were compared by using the $\chi^{2}$ test or the Fisher exact test and McNemar test for paired data and logistic regression analysis. The sensitivity and specificity, PPV, NPV, false-positive rates, and false-negative rates were calculated.

To evaluate interobserver agreement, we calculated the weighted $\kappa$ index with a CI of $95 \%$.

For all tests, a $P$ value $<.05$ was considered statistically significant.

\section{Results}

As mentioned above, 3 patients with their respective 3 nodules were initially excluded from the prospective study because it was not possible to know definite pathologic results. Therefore, 103 patients with a total of 106 thyroid nodules were studied with conventional B-mode sonography and US elastography. Eighty-nine patients were women $(86.4 \%)$ and 14 were men $(13.6 \%)$.

Fifty-two of the 106 nodules (49.1\%) were located in the right lobe of the gland; 49 nodules $(46.2 \%)$, in the left lobe; 5 nodules were sited at the isthmus of the thyroid gland (4.7\%).

All patients underwent FNAB, and the cytologic results of the nodules based on the classification proposed by the Bethesda conference were as follows: $6(5.7 \%)$ nodules corresponded to category 1 (malignant), 4 (3.8\%) nodules were included in category 2 (suspicious for malignancy), 7 nodules $(6.6 \%)$ were classified as category 3 (suspicious for follicular or Hürthle cell neoplasm), 88 nodules (83\%) were benign (category 5$)$, and 1 nodule $(0.9 \%)$ was initially classified as category 6 (insufficient material) and required surgery. No patient from the final series was included in category 4 (atypia of uncertain significance).

On definite pathologic results of the 106 nodules, 10 (9.4\%) nodules corresponded to malignant lesions (8 papillary carcinomas, 1 medullar carcinoma, and 1 anaplastic carcinoma) and 96 nodules $(90.6 \%)$ were benign.

Table 1 shows different variables studied with US elastography in absolute numbers and percentages obtained with consensus from both radiologists and nodule location on the thyroid gland.

In reference to strain, 16 of the total of 106 nodules $(15.1 \%)$ were predominantly rigid, 44 nodules $(41.5 \%)$ were considered predominantly soft, and 46 (43.4\%) lesions were classified as indeterminate if it was not possible to classify them in either of the 2 other groups. Fifteen (14.2\%) of the nodules had a larger size on elastography than on B-mode sonography, 62 nodules were similar in size in both techniques, and 29 lesions $(27.4 \%)$ showed a smaller size with US elastography. Ill-defined or irregular margins were defined in 41 nodules $(38.7 \%)$, whereas the other 65 nodules showed well-defined or regular margins.

Although the number of cases classified as type I (suspicious for benignity) was similar for the 2 readers, reader 1 classified 6 more nodules in type III (suspicious for malignancy) than reader 2 . This last radiologist classified 7 more cases in type II or the indeterminate category. When consensus was obtained, more cases were included in the indeterminate group, and the total number of nodules classified type I or suspicious for benignity was reduced (Fig 2).

Interobserver agreement in the classification of thyroid nodules into the previously defined US elastography groups I, 
Table 1: Frequency in absolute numbers and percentage of different variables studied with US elastography combined with nodule location in the thyroid gland

\begin{tabular}{|c|c|c|c|c|c|}
\hline & $\begin{array}{l}\text { Total of No. } \\
\text { of Nodules }\end{array}$ & $\begin{array}{c}\text { No. of } \\
\text { Nodules } \\
\text { in Left Lobe } \\
\end{array}$ & $\begin{array}{c}\text { No. of } \\
\text { Nodules } \\
\text { in Right Lobe }\end{array}$ & $\begin{array}{l}\text { No. of } \\
\text { Nodules } \\
\text { in the } \\
\text { Isthmus }\end{array}$ & $\begin{array}{c}P \\
\text { Value } \\
\end{array}$ \\
\hline \multicolumn{6}{|l|}{ Elasticity } \\
\hline Predominantly rigid & $16(15.1 \%)$ & $9(56.3 \%)$ & $7(43.7 \%)$ & $0(0 \%)$ & .436 \\
\hline Intermediate & $46(43.4 \%)$ & $18(39.1 \%)$ & $26(56.5 \%)$ & $2(4.4 \%)$ & \\
\hline Predominantly soft & $44(41.5 \%)$ & $22(50 \%)$ & $19(43.2 \%)$ & $3(6.8 \%)$ & \\
\hline \multicolumn{6}{|l|}{ Size } \\
\hline Greater diameter in US elastography than B-mode US & $15(14.2 \%)$ & $7(46.7 \%)$ & $8(53.3 \%)$ & $0(0.0 \%)$ & .138 \\
\hline Same size & $62(58.5 \%)$ & $29(46.8 \%)$ & $32(51.6 \%)$ & $1(1.6 \%)$ & \\
\hline Lesser diameter in US elastography than B-mode US & $29(27.3 \%)$ & $13(44.8 \%)$ & $12(41.4 \%)$ & $4(13.8 \%)$ & \\
\hline \multicolumn{6}{|l|}{ Margins } \\
\hline III-defined or irregular & $41(38.7 \%)$ & $20(48.8 \%)$ & $19(46.3 \%)$ & $2(4.9 \%)$ & .905 \\
\hline Well-defined or regular & $65(61.3 \%)$ & $29(44.6 \%)$ & $33(50.8 \%)$ & $3(4.6 \%)$ & \\
\hline
\end{tabular}

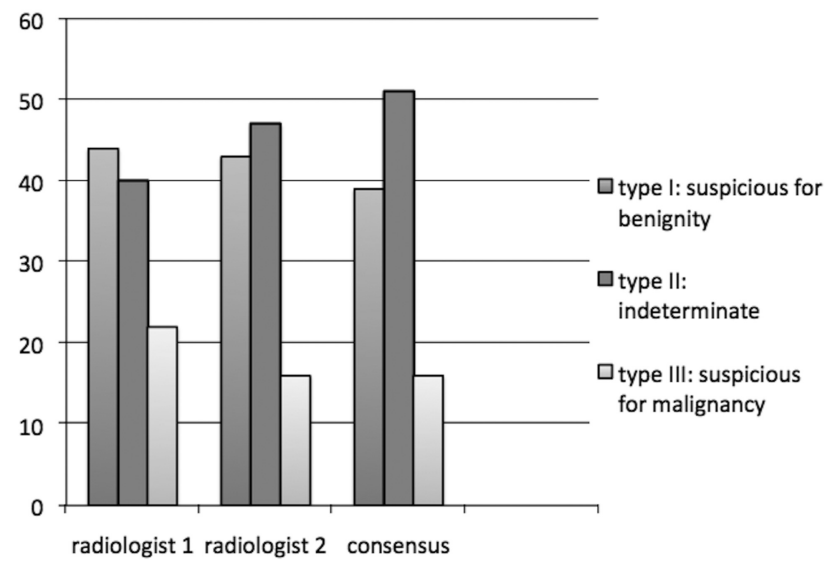

Fig 2. Classification of nodules (absolute numbers) depending on suspicion of malignancy or benignity of lesions with US elastography for each radiologist and by consensus.

II, or III with $P<.001$ was excellent in our study, with a weighted $\kappa$ index of 0.82 (95\% IC, $0.74-0.89$ ).

As a verification of the utility of US elastography, different variables studied with US elastography and defined by consensus between both radiologists correlated with definite pathologic results, considered the reference standard in this study (Table 2).

Six nodules from the group of 10 malignant nodules confirmed by cytology or histology (60\%) were predominantly rigid on US elastography, and the remaining 4 nodules (40\%) showed an indeterminate aspect, which did not allow classifying them as rigid or soft (Fig 3). None of the nodules were described as predominantly soft with US elastography in the group of malignant nodules, and $100 \%$ of the nodules described as soft were benign in the definite pathologic classification (Fig 4).

Regarding nodule size, most studied nodules (62 nodules, $58.5 \%$ ) were similar in size on US elastography and conventional sonography. Fifteen nodules $(14.2 \%)$ had a greater diameter on elastography than on B-mode sonography. Among these last ones, $4(26.7 \%)$ and $11(73.3 \%)$ corresponded to malignant and benign lesions, respectively.

Twenty-nine nodules were smaller on US elastography than with conventional sonography; $96 \%$ of these lesions were benign and $4 \%$ were malignant.

In the evaluation of the utility of the classification of thy-

\begin{tabular}{|c|c|c|c|}
\hline $\begin{array}{l}\text { US Elastography Variables by } \\
\text { Consensus }\end{array}$ & $\begin{array}{l}\text { Malignant } \\
\text { Nodules }\end{array}$ & $\begin{array}{l}\text { Benign } \\
\text { Nodules }\end{array}$ & $\begin{array}{c}P \\
\text { Value }\end{array}$ \\
\hline \multicolumn{4}{|l|}{ Elasticity } \\
\hline Predominantly rigid $(n=16)$ & $6(37.5 \%)$ & $10(62.5 \%)$ & .000 \\
\hline Indeterminate $(n=46)$ & $4(8.7 \%)$ & $42(91.3 \%)$ & \\
\hline Predominantly soft $(n=44)$ & $0(0 \%)$ & $44(100 \%)$ & \\
\hline \multicolumn{4}{|l|}{ Size } \\
\hline $\begin{array}{l}\text { Greater diameter in US } \\
\text { elastography than B-mode } \\
\text { image }(n=15)\end{array}$ & $4(26.7 \%)$ & $11(73.3 \%)$ & .068 \\
\hline $\begin{array}{l}\text { Same size as B-mode image } \\
\qquad(n=62)\end{array}$ & $5(8.1 \%)$ & $57(91.9 \%)$ & \\
\hline $\begin{array}{l}\text { Lesser diameter in US } \\
\text { elastography than B-mode } \\
(n=29)\end{array}$ & $1(3.4 \%)$ & $28(96.6 \%)$ & \\
\hline \multicolumn{4}{|l|}{ Margins } \\
\hline III-defined or irregular $(n=41)$ & $3(7.3 \%)$ & $38(92.7 \%)$ & .548 \\
\hline Well-defined or regular $(n=65)$ & $7(10.8 \%)$ & $58(89.2 \%)$ & \\
\hline
\end{tabular}

roid nodules in groups I (suspicious of benignity), II (indeterminate), and III (suspicious of malignancy) with US elastography, classifications proposed by consensus correlated with pathologic results (Table 3).

Thirty-nine nodules were classified by consensus as type I or suspicious for benignity, and none corresponded to a malignant lesion. On the other hand, among 16 nodules considered by consensus as type III or suspicious for malignancy, 5 nodules corresponded to malignant lesions and 11 nodules were benign. Fifty-one nodules were considered indeterminate in our study, 5 corresponding to malignant lesions and 46 corresponding to benign nodules.

\section{Discussion}

Some pathologic conditions induce considerable changes in the soft-tissue structure, modifying its elastic properties and leading to increased stiffness of the evolved tissue. Because malignancy has been correlated with hardness of tissues, palpation of the thyroid gland constitutes a way of discriminating nodules suspicious for malignancy from those that are benign. This method is subjective and depends on the depth of the lesion.

Elastography is a technique that uses sonography to analyze 


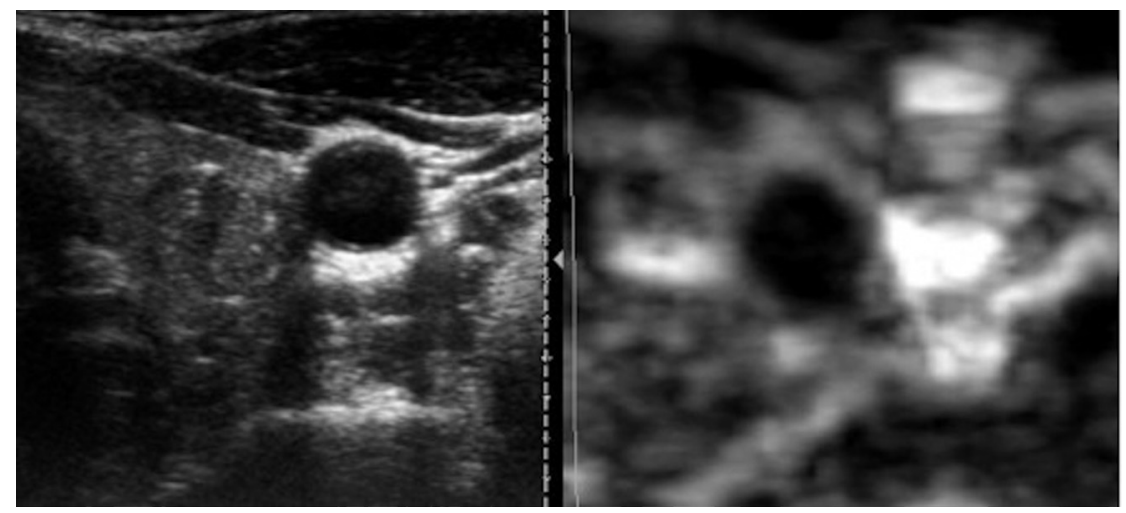

Fig 3. A predominantly hypoechoic nodule with microcalcifications is seen in conventional B-mode sonography image. US elastography image shows a homogeneously black nodule representing a predominantly rigid lesion, sized similar to that on B-mode image. This nodule corresponded to a papillary carcinoma on FNAB and surgery.

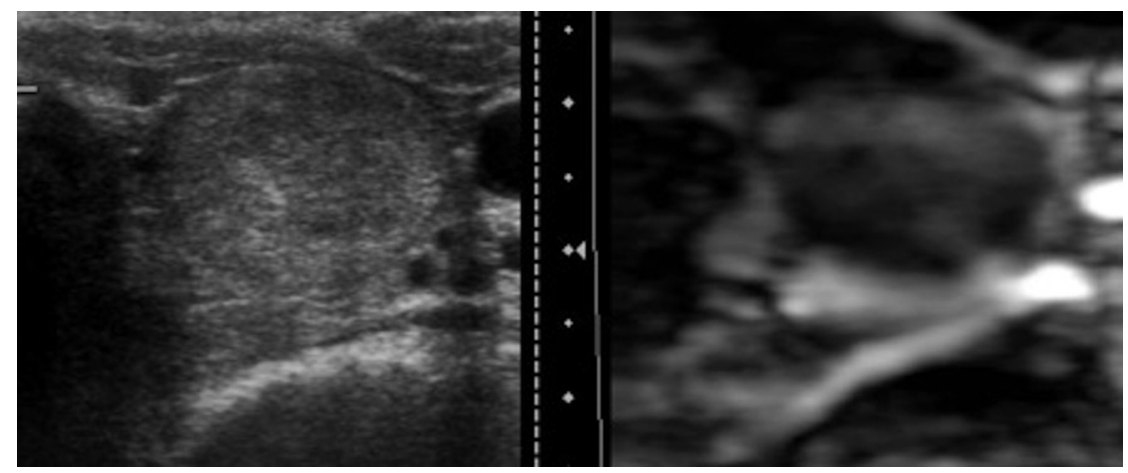

Fig 4. B-mode sonographic and US elastographic images of a benign nodule are shown. A well-defined heterogeneous nodule on conventional sonography shows a predominantly soft pattern of stiffness on the US elastogram.

\begin{tabular}{|c|c|c|c|}
\hline $\begin{array}{l}\text { Classification of Nodules with US } \\
\text { Elastography by Consensus }\end{array}$ & $\begin{array}{c}\text { Malignant } \\
\text { Nodules }\end{array}$ & $\begin{array}{l}\text { Benign } \\
\text { Nodules }\end{array}$ & $\begin{array}{c}P \\
\text { Value }\end{array}$ \\
\hline Type I: suspicious for benignity $(n=39)$ & 0 & 39 & .001 \\
\hline Type II: indeterminate $(n=51)$ & 5 & 46 & \\
\hline Type III: suspicious for malignancy $(n=16)$ & 5 & 11 & \\
\hline
\end{tabular}

the stiffness of a nodule by measuring the amount of distortion that occurs when the nodule is subjected to external pressure. ${ }^{22}$ This technique has been extensively studied in breast lesions and the prostate, pancreas, and lymph nodes. ${ }^{18-21}$ Recent studies have evaluated the use of US elastography for detecting malignant thyroid nodules. ${ }^{23-32}$ These uniformly suggest that US elastography increases the ability to discriminate benign and malignant nodules. Recently, Bojunga et $\mathrm{al}^{31}$ published a meta-analysis of elastography for the differentiation of benign and malignant thyroid nodules, concluding that US elastography can be used with high sensitivity in the work-up of thyroid nodules and might be a useful method in addition to or even instead of FNAB to select patients for surgery.

We present a large series of 103 patients who have been studied with elastography for the evaluation of thyroid nodules. All patients included had been referred for the FNAB procedure as occurs in real clinical practice, so we did not study any nodule that was not previously indicated for aspira- tion. We believe that this method is more representative of the population that will undergo the test in clinical care.

We proposed a simplified classification of stiffness based on gray-scale patterns instead of color because we believe that this method of interpretation is more reliable and reproducible. We also separated images from conventional sonography and US elastography for interpretation to avoid the influence of one reader over another; to our knowledge, only Lyshchik et all ${ }^{26}$ have used this procedure, which we believe is essential for blinded analysis. In addition, we evaluated and compared tumor size with B-mode sonography and US elastography; to our knowledge, only Lyshchik et $\mathrm{al}^{26}$ have included this variable in the analysis.

In our study, there was a significant statistical association between elasticity as an independent variable and the classification proposed (combining elasticity, size, and margins) and definite pathologic results. Malignant nodules could be excluded by elastography (44 nodules appeared soft in US elastography; all were histologically benign) in this cohort of patients. US elastograms predicted malignancy with $100 \%$ sensitivity and $40.6 \%$ specificity. Sensitivity was very high in this study and no false-negatives were found, so all nodules interpreted as suspicious for benignity in our study, mostly due to extensive white areas in gray-scales in elastograms, were benign on pathologic results. This result is concordant with a high NPV of the technique and suggests that US elastography could be helpful in the discrimination of nodules that should be left alone and for which conventional sonographic follow-up would be sufficient. All malignant nodules on definite 
diagnosis were suspicious for malignancy or indeterminate on elastograms in the present study; all those nodules needed to be studied by FNAB to avoid missed malignant lesions. However, in the group of nodules that appeared predominantly rigid, $62.5 \%$ were false-positive. The specificity of the US elastography in our study was a very low, probably secondary to a high number of indeterminately classified nodules, in which determining malignancy or benignity was not possible with elastography because only minor information was provided.

The $\kappa$ index of concordance for elastographic interpretation in our study was 0.82 (95\% CI), reflecting an excellent interobserver agreement. In their published article, Park et $\mathrm{al}^{29}$ did not find a statistically significant concordance.

Most publications referring to US elastography in the evaluation of thyroid nodules propose a scale of tissue stiffness based on the color of the elastograms: 4-point scales, ${ }^{23-26}$ 5 -point scales, ${ }^{28}$ and 6-point scales. ${ }^{24}$ Lyshchik et $\mathrm{al}^{26}$ evaluated 52 thyroid nodules in 31 patients and found a specificity of $96 \%$ and a sensitivity of $82 \%$. Asteria et $\mathrm{al}^{25}$ examined 86 nodules in 67 patients and found a sensitivity of $94 \%$ and a specificity of $81 \%$. The PPVs and NPVs were $55 \%$ and $98 \%$, respectively. Friedrich-Rust et $\mathrm{al}^{23}$ investigated 53 nodules in 50 patients and found an $86 \%$ sensitivity and an $87 \%$ specificity. Rago et $\mathrm{al}^{28}$ found scales $4-5$ highly predictive of malignancy with a sensitivity of $97 \%$ and a specificity of $100 \%$. An off-line processing of strain images, a different method from ours, was also used in the study of Lyshchik et al. Hong et $\mathrm{al}^{24}$ evaluated the diagnostic utility of real-time US elastography with a 6-point scale of color in elastograms of 90 patients who were referred for surgical treatment, so the final diagnosis was based on the results of the histopathologic examination of resected thyroid gland tissue of the 145 thyroid nodules studied. High sensitivity (88\%) and specificity (90\%) were found, with a PPV of $81 \%$ and a NPV of $93 \%$.

Recently, Rago et $\mathrm{al}^{32}$ studied 176 patients with 195 nodules with specifically indeterminate or nondiagnostic cytology on FNAB for whom histology was available after thyroidectomy, and proposed a 3-level elasticity score, similar to the method we used. The score of 1 , describing high elasticity, was strongly predictive of benignity. By combining scores 2 and 3, they found that US elastography had a sensitivity of $96.8 \%$ and a specificity of $91.8 \%$. They also studied interobserver agreement (95\%) but exclusively based on color elastograms. However they did not evaluate the size of the nodule with respect to B-mode conventional sonography or either margin of the nodule.

Some limitations in this study need to be addressed. First, some publications speculate that US-elastograms of nodules with cystic components or coarse calcifications are not reliable, and we have not excluded these nodules from the study. Second, as in other publications, it was not possible in all cases in our study to establish the rigidity of the lesion compared with the surrounding parenchyma, which is the classic proposed optimal interpretation. Previous reports concluded that US elastography and even FNAB are not suitable for the diagnosis of follicular carcinoma, and 4 of 9 follicular carcinomas in the meta-analysis published by Bojunga et $\mathrm{al}^{31}$ were missed on US elastography. We had no case of follicular carcinoma in our series that may have altered the results observed. Further series including more nodules, specifically follicular carcinomas, are required.

\section{Conclusions}

We believe that US elastography is a promising technique that can assist in the differential diagnosis of thyroid cancer and in the selection of nodules suitable for FNAB. To date, US elastography is a relatively easy and quick-to-perform supplemental technique that may be practical for routine clinical care, mostly helping to identify probable benign nodules that could be followed up. US elastography does not require off-line strain imaging reconstruction, and performing it with other techniques (ie, by using shear-waves) will probably increase its overall accuracy. Consequently, we believe that it may be useful to introduce US elastography into routine clinical practice.

\section{References}

1. Tumbridge WM, Evered DC, Hall R, et al. The spectrum of thyroid disease in a community: the Whick-ham Survey. Clin Endocrinol (Oxf) 1997;7:481-93

2. Gilliland FD, Hunt WC, Morris DM, et al. Prognostic factors for thyroid carcinoma: a population-based study of 15,698 cases from the Surveillance, Epidemiology and End Results (SEER) program 1973-1991. Cancer 1997;79: $564-73$

3. Frates MC, Benson CB, Charboneau JW, et al. Management of thyroid nodules detected at US: Society of Radiologists in Ultrasound Consensus Conference Statement. Radiology 2005;237:794-800

4. Tan GH, Gharib H. Thyroid incidentalomas: management approaches to nonpalpable nodules discovered incidentally on thyroid imaging. Ann Intern Med 1997;126:226-31

5. Fish SA, Langer JE, Mandel SJ. Sonographic imaging of thyroid nodules and cervical lymph nodes. Endocrinol Metab Clin N Am 2008;37:401-17

6. Cappelli C, Castellano M, Pirola I, et al. The predictive value of ultrasound findings in the management of thyroid nodules. QJM 2007;100:29-35

7. Kim EK, Park CS, Chung WY, et al. New sonographic criteria for recommending fine-needle aspiration biopsy of nonpalpable solid nodules of the thyroid. AJR Am J Roentgenol 2002;178:687-91

8. Park JY, Lee HJ, Jang HW, et al. A proposal for a thyroid imaging reporting and data system for ultrasound features of thyroid carcinoma. Thyroid 2009;19: 1257-64

9. Sipos JA. Advances in ultrasound for the diagnosis and management of thyroid cancer. Thyroid 2009;19:1363-72

10. Hong YJ, Son EJ, Kim EK, et al. Positive predictive values of sonographic features of solid thyroid nodule. Clin Imaging 2010;34:127-33

11. Moon HJ, Kwak JY, Kim MJ, et al. Can vascularity at power Doppler US help predict thyroid malignancy? Radiology 2010;255:260-69

12. Choi YJ, Yun JS, Kim DH. Clinical and ultrasound features of cytology diagnosed follicular neoplasm. Endocr J 2009;56:383-89

13. Hagag $P$, Strauss $S$, Weiss M. Role of ultrasound-guided fine-needle aspiration biopsy in evaluation of nonpalpable thyroid nodules. Thyroid 1998;8:989-95

14. Baloch ZW, Cibas ES, Clark DP, et al. The National Cancer Institute Thyroid Fine-Needle Aspiration State of the Science Conference: a summation. Cytojournal 2008;5:6

15. Baloch ZW, Livolsi VA, Asa SL, et al. Diagnostic terminology and morphologic criteria for cytologic diagnosis of thyroid lesions: a synopsis of the National Cancer Institute Thyroid Fine-Needle Aspiration State of the Science Conference. Diagn Cytopathol 2008;36:425-37

16. Seo HS, Lee DH, Park SH, et al. Thyroid follicular neoplasms: can sonography distinguish between adenomas and carcinomas? J Clin Ultrasound 2009;37: 493-500

17. Frey H. Realtime elastography: a new ultrasound procedure for the reconstruction of tissue elasticity [in German]. Radiologe 2003;43:850-55

18. Itoh A, Ueno Ei, Thono E, et al. Breast disease: clinical application of US elastography for diagnosis. Radiology 2006;239:341-50. Epub 2006 Feb 16

19. Lyshchik A, Higashy T, Asato R, et al. Cervical lymph node metastases: diagnosis at sonoelastography: initial experience. Radiology 2007;243:258-67. Epub 2007 Feb 9

20. Thomas A, Fischer T, Frey H, et al. Real-time elastography: an advanced method of ultrasound-first results in 108 patients with breast lesions. Ultrasound Obstet Gynecol 2006;28:335-40

21. Cochlin DL, Ganatra RH, Griffiths DF. Elastography in the detection of prostatic cancer. Clin Radiol 2002;57:1014-20

22. Chaturvedi $P$, Insana MF, Hall TJ. Ultrasonic and elasticity imaging to model disease-induced changes in soft-tissue structure. Med Image Anal 1998;2: 325-38 
23. Friedrich-Rust M, Sperber A, Holzer K, et al. Real-time elastography and contrast-enhanced ultrasound for the assessment of thyroid nodules. Exp Clin Endocrinol Diabetes 2010;118:602-09

24. Hong Y, Liu W, Li Z, et al. Real-time ultrasound elastography in the differential diagnosis of benign and malignant thyroid nodules. J Ultrasound Med 2009;28:861-67

25. Asteria C, Giovanardi A, Pizzocaro A, et al. US elastography in the differential diagnosis of benign and malignant thyroid nodules. Thyroid 2008; 18:523-31

26. Lyshchik A, Higashi T, Asato R, et al. Thyroid gland tumor diagnosis at US elastography. Radiology 2005;237:202-11

27. Dighe M, Bae U, Richardson ML, et al. Differential diagnosis of thyroid nodules with US elastography using carotid artery pulsation. Radiology 2008;248: $662-69$
28. Rago T, Santini F, Scutari M, et al. Elastography: new developments in ultrasound for predicting malignancy in thyroid nodules. J Clin Endocrinol Metab 2007;92:2917-22

29. Park SH, Kim SJ, Kim E-K, et al. Interobserver agreement in assessing the sonographic and elastographic features of malignant thyroid nodules. $A J R$ Am J Roentgenol 2009;193:416-23

30. Rago T, Scutari F, Santini V, et al. Real-time elastosonography: useful tool for refining the presurgical diagnosis in thyroid nodules with indeterminate or nondiagnostic cytology. J Clin Endocrinol Metab 2010;95:5274-80

31. Bojunga J, Herrmann E, Meyer G, et al. Real-time elastography for the differentiation of benign and malignant thyroid nodules: a meta-analysis. Thyroid 2010;20:1145-50

32. Rago T, Vitti P. Potential value of elastosonography in the diagnosis of malignancy in thyroid nodules. Q J Nucl Med Mol Imaging 2009;53:455-64 